\title{
Los antibioticos no fueron efectivos en la sinusitis aguda
}

van Buchem F L, Knottnerus J A, Schrijnemaekers V J J y col. Primary-care-based randomised placebo-controlled trial of antibiotic treatement in acute maxillary sinusitis.

Lancet $1997 ; 349: 683-7$

\section{Objetivo}

Determinar la efectividad del tratamiento con antibióticos en pacientes con sospecha de sinusitis o radiografía anormal que consultan a médicos generales.

\section{Lugar}

Departamento de Otorrinolaringología, Microbiología, y Medicina General en un hospital de Holanda.

\section{Diseño}

Ensayo clínico randomizado, doble ciego, controlado con placebo. Un año de seguimiento.

\section{Pacientes}

Se incluyeron 214 pacientes con sospecha clínica de sinusitis y radiografía de senos anormal. Los criterios de exclusión fueron: otras enfermedades nasales (ej: pólipos), bronquitis concomitante, síntomas por más de 3 meses, tratamiento antibiótico durante el último mes, contraindicaciones para recibir amoxicilina; radiografías normales y presencia de pólipos o aumento de la mucosa menor de $5 \mathrm{~mm}$.

\section{Intervención}

Los pacientes fueron tratados con inhalaciones mentoladas, paracetamol a demanda y randomizados para amoxicilina $750 \mathrm{mg}$. 3 veces/día por 7 días $(\mathrm{n}=108)$ o placebo en la misma frecuencia $(n=106)$. Los pacientes fueron evaluados luego de 1 y 2 semanas. En la segunda entrevista se realizó una nueva radio- grafía, evaluada por el mismo radiólogo ciego al tratamiento. Si los síntomas requerían nuevos tratamientos luego de 2 semanas se realizaba una punción del seno maxilar. Los pacientes eran instruidos para consultar a su médico en caso de recurrencias.

Medición de resultados principales

El resultado principal a medir fue la tasa de curación a las 2 semanas y la escala de síntomas a la semana y a las 2 semanas.

Resultados principales

El análisis se realizó por intención de tratar. La media de duración del período sintomático previo a la intervención fue 2.2 semanas. Los 2 grupos estuvieron bien balanceados en edad, sexo, síntomas y examen físico. Luego de 2 semanas no hubo diferencias significativas en la curación (65\% grupo Atb vs $53 \%$ grupo placebo), en la disminución de los síntomas ( $83 \%$ vs $78 \%$ ). Las radiografías a los 14 días fueron normales en el $74 \%$ del grupo Atb. y en el $60 \%$ del grupo placebo $(p=0.03)$. La adherencia al tratamiento fue de un 98\%. El 28\% del grupo Atb. y el $9 \%$ del grupo placebo reportaron efectos adversos menores $(\mathrm{p}=0.001)$. No hubo diferencias en la tasa de recaídas (21\% Atb. y $17 \%$ placebo).

\section{Conclusiones}

En este estudio los antibióticos no mejoran el curso de la sinusitis aguda en los pacientes atendidos por médicos generales. Para estos pacientes, la radiografía no resulta necesaria y el manejo inicial puede limitarse al tratamiento sintomático. Si los antibióticos son necesarios en casos más severos deberá establecerse en otros estudios.

\section{Comentario}

Los resultados de este trabajo nos conducen a la práctica, ya evaluada con anterioridad, del tratamiento sintomático en casos de sinusitis aguda leve a moderada.

De todos modos algunas preguntas podríamos establecer:¿ cuántos de estos pacientes interpretados como sinusitis aguda reunen criterios clínicos de rinitis o resfrío común? Algunos trabajos han demostrado que existe algún grado de inflamación en la mucosa de los senos paranasales en pacientes con rinitis o resfrío común(1). ¿Es la radiografía de senos paranasales de utilidad en la evaluación de una sinusitis aguda? Los autores concluyen que no y existen datos que sugieren que el valor predictivo de la historia clínica y del examen físico permiten descartar el diagnóstico de sinusitis (2). Los exámenes complementarios estarían indicados cuando manejamos pacientes con probabilidades intermedias de sinusitis. En este estudio el $20 \%$ de los pacientes no fueron derivados por los médicos generales pues consideraron que no estaban lo suficientemente enfermos para recibir antibióticos; esto refuerza las conclusiones de los autores ya que incluían solo aquellos que eran clínicamente más sintomáticos.(Ver Glosario: sesgo de selección)

En este trabajo se utilizó la amoxicilina como tratamiento y los autores aclaran que el Haemophilus influenzae productor de B lactamasa (HIB) tiene un $5 \%$ de prevalencia en esa región y la respuesta al tratamiento de 7 días ya había sido evaluada. Nosotros debiéramos considerar las resistencias antibióticas que predominan en nuestro área de trabajo al elegir el antibiótico a utilizar. (Ver Glosario: validez externa). En las áreas en donde el HIB sea prevalente y uno decida utilizar antibióticos, la combinación de trimetoprima-sulfametoxazol es una buena elección por cobertura y costo.

\section{Nora Gimpel Unidad de Medicina Familiar y Preventiva Hospital Italiano de Buenos Aires}

1.Gwaltney JM, Phillips CD, Miller RD. Computed tomographic study of the common cold. N Engl J Med

1994; 330:25-30.

2. van Buchem M, Peeters MF, Beaumont JF. Acute maxilary sinusitis in general practicethe relation between clinical picture and objective findings.

Eur J Gen Pract 1995;1:155-60 